DID: An empirical overview

\title{
Dissociative Identity Disorder: An empirical overview
}

\author{
Martin J Dorahy ${ }^{1}$, Bethany L Brand ${ }^{2}$, Vedat Şar $^{3}$, Christa Krüger ${ }^{4}$, Pam Stavropoulos ${ }^{5}$, \\ Alfonso Martínez-Taboas ${ }^{6}$, Roberto Lewis-Fernández ${ }^{7}$ and Warwick Middleton ${ }^{8}$
}

RUNNING HEAD: DID: An empirical overview

${ }^{1}$ Department of Psychology, University of Canterbury, New Zealand

${ }^{2}$ Department of Psychology, Towson University, USA

${ }^{3}$ Department of Psychiatry, Istanbul University, Turkey

${ }^{4}$ Department of Psychiatry, University of Pretoria, South Africa

${ }^{5}$ Adults Surviving Child Abuse, Australia

${ }^{6}$ Department of Psychology, Carlos Albizu University, Puerto Rico

${ }^{7}$ Department of Psychiatry, Columbia University, USA

${ }^{8}$ Department of Psychiatry, University of Queensland, Australia

Corresponding author: Martin J Dorahy, Department of Psychology, University of Canterbury, Private Bag 4800, Christchurch, 8140, New Zealand

Email: martin.dorahy@canterbury.ac.nz

\begin{abstract}
Objective: Despite its long and auspicious place in the history of psychiatry, dissociative identity disorder (DID) has been associated with controversy. This paper aims to examine the empirical data related to DID and outline the contextual challenges to its scientific investigation. Method: The overview is limited to DID-specific research in which one or more of the following conditions are met: 1) a sample of participants with DID was systematically investigated, 2) psychometrically-sound measures were utilised, 3) comparisons were made with other samples, 4) DID was differentiated from other
\end{abstract}


disorders, including other Dissociative Disorders, 5) extraneous variables were controlled or 6) DID diagnosis was confirmed. Following an examination of challenges to research, data is organised around the validity and phenomenology of DID, its aetiology and epidemiology, the neurobiological and cognitive correlates of the disorder, and finally its treatment. Results: DID was found to be a complex yet valid disorder across a range of markers. It can be accurately discriminated from other disorders, especially when structured diagnostic interviews assess identity alterations and amnesia. DID is aetiologically associated with a complex combination of developmental and cultural factors, including severe childhood relational trauma. The prevalence of DID appears highest in emergency psychiatric settings and affects approximately $1 \%$ of the general population. Psychobiological studies are beginning to identify clear correlates of DID associated with diverse brain areas and cognitive functions. They are also providing an understanding of the potential metacognitive origins of amnesia. Phase-oriented empirically-guided treatments are emerging for DID. Conclusions: The empirical literature on DID is accumulating, although some areas remain under-investigated. Existing data show DID as a complex, valid, and not uncommon disorder, associated with developmental and cultural variables, that is amenable to psychotherapeutic intervention.

\section{Keywords}

Dissociative identity disorder, validity, phenomenology, aetiology, psychobiology, treatment 
Dissociative identity disorder (DID) has an auspicious place in the archives of psychiatry. It captured the attention of many of the great $19^{\text {th }}$ and early- $20^{\text {th }}$ century thinkers, whose ideas form the foundation of modern psychiatric thought (James, 1896 [see Taylor, 1983], Janet, 1907; Prince, 1905). More recently DID has become the subject of considerable debate (e.g., Dalenberg et al., 2012; Gleaves, 1996; McHugh and Putnam, 1995; Merskey, 1992), especially around its validity, aetiology and prevalence. Often overlooked is the empirical understanding of DID accrued over 30 years, and which commenced in earnest with the adoption of DID (then referred to as Multiple Personality Disorder) as a discrete diagnostic entity in the third edition of the Diagnostic and Statistical Manual of Mental Disorders (DSM-III; American Psychiatric Association, 1980). The accumulation of empirical knowledge paints a clear and consistent picture of DID.

This overview is designed to provide a current 'broad-brush' outline of the scientific foundation of DID by focusing on DID-specific research. Thus the overview excludes opinion pieces and papers without DID data and is confined to studies identified in searches of major psychological (e.g., Psycinfo) and psychiatric databases (e.g., Medline) which investigated individuals with DID where one or more of the following conditions were met: 1) a sample of participants with DID was systematically investigated, 2) psychometrically-sound measures were utilised, 3) comparisons were made with other samples, 4) DID was differentiated from other disorders, including other Dissociative Disorders ${ }^{1}, 5$ ) extraneous variables were controlled or 6) DID diagnosis was

\footnotetext{
${ }^{1}$ Some studies on DID and dissociative disorder not otherwise specified (DDNOS)type 1 , a condition closely resembling DID, were included.
} 
confirmed (e.g., with structured interview). Sociological and contextual issues, especially with reference to the scientific study of DID, are explored.

We have chosen to limit the current overview to empirical research on DID, thereby bypassing a wide literature on dissociation and dissociative disorders. This literature provides abundant, consistent evidence that dissociative experiences, symptoms and disorders exist throughout the world (Spiegel et al., 2013). Stein et al. (2013) found dissociative symptoms among 14.4\% of individuals with PTSD from a sample of 25,018 respondents from 16 different countries. In a review of the cross-cultural assessment of dissociation, Lewis-Fernández et al. (2007) provide extensive evidence that reliably assessed dissociative symptoms and disorders are found in many different countries.

Limiting the overview to DID data precludes important discussions about the commonalities among the dissociative disorders and the conceptual nature of dissociation (e.g., whether it is best conceived as a continuum, as a set of discrete categories or as a combination of these). Recent reviews examine these and other relevant issues (e.g., Dalenberg et al., 2012; Spiegel et al., 2013). Our aim is to provide an up-to-date overview of scientific evidence about DID by reviewing the most compelling research in a variety of areas, including DID's construct validity, aetiology, prevalence, psychobiological and cognitive foundations, and treatment. Challenges in the empirical investigation of DID will first be considered, to provide the contextual landscape for the work that follows. Each section might fruitfully be reviewed in depth following this broad overview. 
DID: An empirical overview

\section{'Contextual Challenges in Empirical Investigation of DID}

Research on DID is constrained by obstacles atypical for those of other psychiatric disorders. The impediments cover five areas: diagnostic concerns, cultural issues, post-traumatic avoidance, cost-benefit issues, and conceptual challenges.

Diagnostic concerns

DID is only weakly represented in the $10^{\text {th }}$ edition of the International Classification of Diseases (ICD-10; among "other" dissociative disorders). In the DSM-5 it is more fully elaborated. The discrepancy of definition hampers international research efforts. DID patients usually present a plethora of diverse symptoms in addition to core diagnostic features (Şar and Ross, 2006; see 'construct validity', below). This polysymptomatic profile may obscure DID unless dissociative symptoms are systematically assessed. Since major general psychiatric diagnostic instruments used in epidemiological and clinical research (e.g., the Structured Clinical Interview for DSM-IV [SCID] and the Composite International Diagnostic Interview [CIDI]) lack a Dissociative Disorders (DD) section (Şar and Ross, 2009), DID is repeatedly under-researched. However, many researchers outside the field of dissociation are now including questions about dissociation, which may lead to increased assessment for DD, including DID, more widely. Adding DID symptoms to existing diagnostic and screening tools, and developing shorter diagnostic instruments specific for DID is crucial.

\section{Cultural issues}

Cultural variation in the clinical manifestation of DID remains under-researched (see 'Aetiological Pathways and Influences in the Development of DID', below). Challenges that have contributed to the paucity of cross-cultural research include lack of 
DID: An empirical overview

uniformity between international diagnostic classifications (ICD and DSM), and the difficulty of assessing for diverse modes of dissociative self-representation (e.g., different idioms in different regions can preclude meaningful comparative research).

A further contextual challenge to DID research pertains to the varying nature of identity across cultures, that 'identity' per se may not be unified, and that 'self' is constructed as more relational in some contexts and cultures than in others (Castillo, 1997). Whereas the 'Western' conception of self emphasises autonomy, DID challenges the notion of identity as fixed, unitary and autonomous. Thus it is not surprising that identity-related cultural differences complicate comparative DID research.

\section{Post-traumatic avoidance}

DID is consistently linked to childhood relational trauma (see 'Aetiological Pathways and Influences in the Development of DID', below) and post-traumatic avoidance operates at several levels, both individually and socially. Many DID patients are conditioned 'not to tell' of their trauma, which pertains to intra-psychic factors (selfdenial, shame), threats from perpetrators, and/or experiences of being disbelieved. This reticence may hinder their participation in research studies.

A major challenge in researching DID relates to the reaction of the human mind when confronted with terrible, unspeakable events directed at children - that is, defensive denial of their occurrence or minimisation of their severity. Such denial, to which researchers, clinicians and policy makers are also subject (Herman, 1992), sabotages understanding and effective treatment of the impact of such events on victims.

The abuse of adult power over children (which violates the central societal norm of protection of the young) calls into question such mainstream social institutions as the 
DID: An empirical overview

family and other organisations which ostensibly operate in the provision of care. Since the aetiology of DID is associated with childhood relational trauma, the discomfort caused by studying DID may serve as a potent disincentive to its investigation. Thus avoiding study of DID protects mainstream social institutions - at the expense of the children who are violated by them - as well as enabling researchers, clinicians, and the public to retain a comforting denial of both the occurrence of abuse and its disabling psychiatric legacy. Hence avoidance of the central issues associated with DID operates not only in the patient, but in society at large.

\section{Cost-Benefit Issues}

Further challenges to DID research include the expensive treatment for these complicated, heterogeneous patients, and lack of funding for both long-term treatment and the long-term research needed to study treatment outcome. The cost of DID to health systems and its amenability to treatment remain largely outside the awareness of researchers, clinicians and policy makers. Thus DID is not targeted as a research priority in mental health.

As neurobiological studies on DID accrue, they show that DID is as suitable for biological investigation as any other psychiatric disorder (see 'Unique neurophysiological profile of DID'). But as DID shows only limited responsiveness to existing medication, it falls outside the purview of many researchers who focus on disorders that respond better to pharmacotherapy and short-term treatments, and that are diagnosed by current standardised interviews. 
DID: An empirical overview

Conceptual challenges

Other contextual challenges relate to the concept of self as an autonomous and integrated entity (which is challenged by the psychic fragmentation of DID), and the limits of objectivity and neutrality when addressing the enormity of psychological trauma associated with DID. Conceptual and methodological challenges include the risk of abstraction of symptomatology from its social context, the discounting of lived experience as a form of evidence (and corresponding need for phenomenological approaches) and the reductionism of standard classificatory nomenclature as accurate representation of complex dimensions of subjectivity: "At issue here are core questions about what constitutes the appropriate data upon which to base our understandings of mental life" (Hornstein, 2013:31).

\section{Validity and phenomenology of DID}

The validity of psychiatric disorders is established by demonstrating content validity (i.e., a consistent, detailed clinical presentation found by independent researchers), criterion validity (i.e., data from laboratory, psychological and neurobiological tests must be consistent with the clinical presentation), and construct validity (the disorder can be distinguished from other disorders and from simulation; Robins and Guze, 1970). Data support all three types of validity for DID (Gleaves et al., 2001). 
DID: An empirical overview

Content Validity: Repeated, detailed, independently-observed clinical presentation.

The dissociative symptoms of identity confusion, identity alteration and amnesia ${ }^{2}$ form the core symptoms differentiating DID from other disorders in the DSM-5, with only the latter two required in ICD-10 (APA, 2013; World Health Organization [WHO], 1993). While common among individuals with DID, derealisation and depersonalisation ${ }^{3}$ are not required for the diagnosis (APA, 2013; Steinberg et al., 1994a). Researchers from Asia, North and South America, Europe, and Australia have found these five dissociative symptoms are typically present in DID, often at severe levels (e.g., Boon and Draijer, 1993a; Gingrich, 2009; Martínez-Taboas, 1991; Middleton and Butler, 1998; see 'construct validity' section for further discussion). The consistent clinical picture across cultures and research laboratories supports the content validity of DID.

Criterion Validity: Consistency across multiple methods of assessment

The structured clinical interviews for diagnosing DID show inter-rater reliability rates that are as high, and generally higher, than those for other psychiatric disorders (e.g., .80 or higher for the Structured Clinical Interview for DSM-IV Dissociative Disorders [SCID-D; Steinberg, Rounsaville and Cicchetti, 1990; See Gleaves et al., 2001]). The SCID-D/SCID-D-Revised (Steinberg, 1994a; 1994b; Steinberg et al., 1990) assesses five categories of dissociative symptoms (identity confusion, identity alteration, amnesia, depersonalisation, derealisation) and allows diagnosis of DID. For example,

2 Identity confusion is the subjective sense of conflict or uncertainty about one's identity due to non-integrated or fragmented self-states; identity alteration refers to the objective behaviours that are observable manifestations of different identities; amnesia is an inability to recall autobiographical information. (Steinberg, 1994a). 3 The DSM-5 defines depersonalisation as "experiences of unreality or detachment from one's self" and derealisation as "experiences of unreality or detachment from one's surroundings"' (p. 291). 
DID: An empirical overview

Dutch researchers using the SCID-D found excellent inter-rater reliability for symptom severity scores as well as the presence of a DD, including DID (weighted kappas ranging from .85-.98; Friedl and Draijer, 2000). Scientists in numerous countries have found the SCID-D effective in detecting DID (e.g., Gingrich, 2009; Mueller-Pfeiffer et al., 2013; Ross et al., 2002).

Another structured interview, the Dissociative Disorders Interview Schedule (DDIS; Ross et al., 1989b) assesses the symptoms of the five DSM-IV dissociative disorders, and has good reliability and validity. For example, in detecting DID and dissociative disorder not otherwise specified (DDNOS), the DDIS shows good inter-rater reliability with a clinical interview (kappa=.71), the self-report Dissociative Experiences Scale (DES; Bernstein and Putnam, 1986) taxon (i.e., an empirically derived subscale that distinguishes individuals with a high probability of having a DD from those with other disorders and controls; kappa=.81; [Waller et al., 1996]), and the SCID-D (kappa=.74; Ross et al., 2002).

The DES is an effective screening tool for DID and DDNOS-I (a presentation with dissociative identities but without amnesia), although there appear to be cultural differences in the most effective cut-off scores for adequate sensitivity and specificity (Mueller-Pfeiffer et al., 2013). This may be due to differences in reporting and experiencing dissociation in different countries, and differences in translated versions of the DES and research methodology (Mueller-Pfeiffer et al., 2013). Such findings highlight the importance of determining dissociation scale norms within specific cultural settings. 
DID: An empirical overview

Construct Validity - Discriminant type: Distinctiveness from other disorders

DID can be distinguished accurately from other psychiatric disorders and nonpatients using structured interviews and self-report measures of dissociation. Two core symptoms (identity alteration, amnesia) differentiate DID from other disorders (Steinberg, 1994a). So, too, does the combined frequency of other dissociative symptoms, including identity confusion, depersonalisation/derealisation, and somatoform dissociation (Dell, 2006; Nijenhuis et al., 1999).

The dissociative symptoms in DID and DDNOS appear to be qualitatively different (e.g., identity alteration, amnesia) from other kinds of dissociation (Putnam et al., 1996; Rodewald et al., 2011a). This suggests that assessing a range of dissociative symptoms facilitates differential diagnosis. The core symptoms of DID (identity alteration, amnesia) contribute considerably to detriments in global functioning beyond other dissociative symptoms (e.g., depersonalisation) and Axis I symptoms (MuellerPfeiffer et al., 2012).

The severity and breadth of multiple dissociative symptoms, particularly the pathognomonic symptoms of identity alteration and amnesia, are characteristic of DID. Despite these classic indicators of DID, multiple covert dissociative (e.g., flashbacks, auditory hallucinations) and non-dissociative (e.g., affective instability) symptoms may obscure from clinical view the true nature of the pathology, thereby delaying accurate diagnosis of DID (Rodewald et al., 2011b; Ross and Ness, 2010; Ross et al., 1990a). Research shows, however, that careful assessment of the range of dissociative symptoms can accurately distinguish DID. 
Psychotic and dissociative disorders show symptoms that resemble each other, including most of the Schneiderian symptoms (Kluft, 1987; Ross et al., 1990a; Welburn et al., 2003). For example, studies show that individuals with DID have auditory hallucinations emanating from both inside and outside the head, not unlike in schizophrenia (Dorahy et al., 2009; Honig et al., 1998). Yet patients with DID are more likely to hear more than two voices, including those of children and adults, beginning before 18 years of age (Dorahy et al., 2009).

DID patients do not have true delusions (e.g., they tend not to endorse delusional perception; Kluft, 1987). Patients with DID or allied forms of DDNOS have better cognitive insight than patients with schizophrenia, and similar levels compared to those with obsessive-compulsive disorder or depression (Şar et al., 2012). Dissociative patients also have self-reflective capacities indicating cognitive insight in the non-psychotic range (Brand et al., 2009c; Şar et al., 2012).

Patients with DID may decompensate to a dissociative psychosis as a transient crisis state which may be confused with schizophrenia (Tutkun, Yargic, and Şar, 1995). Patients with such a dissociative (formerly called hysterical) psychosis (Van der Hart et al., 1993) may appear functionally "psychotic" due to temporarily poor reality-testing and disorganised behaviour. The aetiology of the process is post-traumatic and dissociative (e.g., post-traumatic content may manifest in hallucinatory symptoms; Şar and Öztürk, 2009). On the other hand, some patients with a schizophrenic disorder may present with symptoms associated with DID,, thereby fitting the proposed dissociative subtype of schizophrenia (Ross, 2004). Alternatively, this presentation may be due to comorbidity between schizophrenia and DID among traumatised individuals (Şar et al., 2010). 
DID: An empirical overview

No study to date has found DID without multiple non-dissociative comorbid psychopathology (e.g., Boon and Draijer, 1993a; Mueller-Pfeiffer et al., 2012; Rodewald et al., 2011b). Depression and associated symptoms (lability, suicidal ideation) are among the most frequent (e.g., Ellason et al., 1996; Middleton and Butler, 1998). Posttraumatic stress disorder (PTSD) is present in the majority of cases (e.g., Boon and Draijer, 1993a; Middleton and Butler, 1998; Vermetten et al., 2006). Of the anxiety disorders, panic disorder is the most common and generalised anxiety disorder is the least common (Rodewald et al., 2011b). Increased comorbid anxiety disorders may differentiate DID from other conditions, including borderline personality disorder (BPD) and schizophrenia (Fink and Golinkoff, 1990).

Self-harm and substance abuse are typically found in over $50 \%$ of people with DID (e.g., Boon and Draijer, 1993a; McDowell et al., 1999). Over a third have eating or somatoform disorders (Ellason et al., 1996). BPD is the most common personality disorder, and is typically present in between a half to two-thirds of cases (Ellason et al., 1996; Horevitz and Braun, 1984; Middleton and Butler, 1998), with some studies reporting higher rates (Lipsanen et al., 2004; Şar et al., 2003). Crisis states prompting emergency service visits in DID include self-mutilation, flashbacks, non-epileptic seizures and suicide attempts. They also include acute episodes of mixed dissociative and psychotic symptoms characterised by a "revolving door" (rapid switching among identities) or "co-consciousness" (temporary breakdown of internal dissociative barriers) crises (Tutkun et al., 1995). The interplay between psychotic and dissociative processes requires further empirical investigation in these crisis episodes. 
This complex clinical picture complicates assessment and diagnosis. The empirical phenomenological literature (which can be used to assess discriminate validity) suggests that dissociative symptoms, as measured by instruments such as the SCID-D or the DDIS, differentiate DID from other disorders (e.g., Ross et al., 1989a; Welburn et al., 2003). While individuals with DID present a multifaceted symptom profile that goes beyond the dissociative domain, neither personality measures (e.g., Minnesota Multiphasic Personality Inventory-2 [MMPI-2]; Millon Clinical Multiaxial Inventory-III) nor non-dissociative symptom measures reliably differentiate DID from other disorders (e.g., Kemp et al., 1988; Welburn et al., 2003). One exception in the personality domain, however, may be projective tests (e.g., Rorschach) which detect some differences, including those with DID having a greater capacity to develop a working therapeutic alliance (e.g., Brand et al., 2009c).

Some psychiatric patients consciously or unconsciously imitate DID (Draijer and Boon, 1999). Thus an important method of establishing construct validity is determining whether simulators who are knowledgeable about a disorder can imitate it on psychological and neurobiological tests. A growing evidence base using both types of tests indicates that genuine DID can be distinguished from feigned (i.e., malingered, factitious or simulated) DID. The dissociative interviews show the most utility in this differential diagnosis, although some personality tests are also useful. Most self-report measures of dissociation are not effective for this purpose because they do not have validity scales. The SCID-D is effective in distinguishing genuine DID from malingered and factitious DID (Draijer and Boon, 1999; Friedl and Draijer, 2000). Welburn and 
colleagues (2003) found a 0\% false positive rate in distinguishing feigned DID from DID patients using the SCID-D-R.

Psychological tests often include "fake bad" validity scales that consist of items typically endorsed by individuals who are exaggerating symptoms of mental illness. However, research shows that many such validity scales contain items characteristic of the symptoms experienced by traumatised individuals, including those with DID. Thus, paradoxically, they may be endorsed by individuals who are not feigning or exaggerating mental illness. For example, a study compared the MMPI-2 profiles (Butcher et al., 2001) of 53 DID patients with 67 uncoached and 77 coached DID simulators. Monetary awards were given to those who best feigned DID following hours of training about DID, including media and internet information about the disorder (Brand and Chasson, in press). The DID group's scores were more extreme than many psychiatric groups' scores on validity and clinical scales, but they were not more extreme than those found among PTSD or child sexual abuse groups. Furthermore, the direction of the correlations between dissociation scores and the MMPI-2 validity and clinical scales were in the opposite direction for the simulators compared to the DID group for 15 out of 18 correlations conducted.

The researchers concluded that the DID group's elevations on the validity scales stemmed from their endorsement of dissociative and trauma-related items (which are mistakenly included on these scales). For example, one "fake bad" validity item, in abbreviated form, asks participants whether they "Sometimes do things and don't remember doing them" (i.e., dissociative amnesia typical of DID). Another inquires about whether individuals "Feel things aren't real" (i.e., derealisation) (MMPI@-2 
DID: An empirical overview

Booklet of Abbreviated Items) $)^{4}$. Despite these problems with the test's items, in a discriminant function analysis, $83.0 \%$ of simulators and $86.0 \%$ of the DID cases were correctly classified on the MMPI-2 (Brand and Chasson, in press). That is, despite media exposure, training, and incentives, the feigners still could not accurately imitate DID.

Studies using a well-established forensic interview for assessing feigned mental illness, the Structured Interview of Reported Symptoms (SIRS or SIRS-2; Rogers et al., 2010) indicate that if a Trauma Index is used, feigners can be distinguished from DID patients with overall diagnostic power (ODP) as high as 83.3 (Brand et al., 2006; Brand, Tursich, Tzall \& Loewenstein, in press). The Trauma Index is an empirically-derived index of subscales that accurately classifies severely traumatised individuals, because unlike some SIRS/SIRS-2 subscales, its subscales do not include dissociative and traumarelated items. Without the Trauma Index, the overall utility of the SIRS/SIRS-2 is lower (i.e., $\mathrm{ODP}=58.7-81.0$; Brand et al. in press).

Dissociative items are often included on other tests' validity and clinical scales, including the Personality Assessment Inventory's (PAI) NIM scale (a so-called "fake bad" scale; Morey, 1991). Thus it is not surprising that DID individuals show elevated ratings on validity scales that include dissociative items. Yet DID individuals do not elevate on the PAI's validity subscales that do not include dissociative, trauma-related items (Brand, Stadnik and Savoca, 2013). Importantly for the validity of the diagnosis, DID individuals do not typically score above ranges found in other trauma samples,

${ }^{4}$ MMPI-2 Booklet of Abbreviated Items. Copyright (C) 2005 by the Regents of the University of Minnesota. All rights reserved. Used by permission of the University of Minnesota Press. "MMPI" and "Minnesota Multiphasic Personality Inventory" are registered trademarks owned by the Regents of the University of Minnesota. 
particularly if the "fake bad" scales do not include trauma and dissociation content (Brand and Chasson, in press; Brand et al., in press). These data underscore the importance of assessors being informed about research regarding severely dissociative clients to avoid misclassification of those with DID as malingering, exaggerating, or as suffering from a psychotic disorder (i.e., when their pattern of symptoms is in fact characteristic of DID).

Consistent with the psychological tests, neurobiological studies have shown that DID can be accurately differentiated from simulated DID. Reinders and colleagues distinguished DID patients from DID simulators - even simulators high in suggestibility on emotional arousal, cerebral brain flow patterns, heart rate, heart rate variability, and blood pressure (Reinders et al., 2012). Dissociative identities fully aware of trauma experiences showed different subjective, neural, and psychophysiological patterns when listening to autobiographical trauma scripts, compared to dissociative identities who were less aware of trauma experiences. These patterns could not be replicated by simulators, regardless of whether they were high or low in suggestibility (see Schlumpf et al., 2013 for similar findings using a different methodology).

In summary, DID is a disorder that: 1) has a complex clinical presentation; 2) can be discriminated reliably from other disorders according to frequency and severity of multiple dissociative symptoms; and 3) meets accepted standards for content, criterion, and construct validity. Therefore, data consistently indicate DID is a valid diagnosis. 
DID: An empirical overview

Aetiological Pathways and Influences in Development of DID: Cultural and

\section{Relational Context}

There is wide consensus that the processes and mechanisms intrinsic to the experience of psychopathology are sensitive to cultural and societal influences (Eshun and Gurung, 2009). Culture impacts how individuals display and communicate their symptoms, how such symptoms are interpreted, and what type of care is sought. For example, data support the role of culture in patterning the presentation of eating disorders (Anderson-Fye and Becker, 2004), personality disorders (Mulder, 2012), depressive disorder (Korman and Molina, 2010), schizophrenia (Stompe and Friedmann, 2007), and anxiety disorders (Lewis-Fernández et al., 2010).

Both universal and cultural processes influence the development and phenomenology of DID (Dorahy, 2001a). Dissociation and dissociative disorders (DD) can be found in all cultural settings (e.g., Spiegel et al., 2013; Stein et al., 2013). DID has been documented in Turkey, Puerto Rico, Scandinavia, Japan, Canada, Australia, the United States, Philippines, Ireland, United Kingdom, and Argentina, among many other cultural and geographical contexts (Rhoades and Şar, 2005).

DID is intrinsically related to experiences of self and personhood. This point is of particular importance, because Western views of the person emphasise a conception of self as separate, autonomous, self-contained and independent (Cross and Markus, 1999). In a recent review of the role of culture in construction/s of self, Markus and Kitayama (2010) assert that selves actively engage in a dynamic process in which they influence and are influenced by their sociocultural contexts. Western preoccupation with individualism leads to experiences of self as separate or independent from those of others. 
In contrast, non-Western societies tend to endorse an interdependent self, which fosters experiences of self as entwined with the expectations and needs of others.

The cultural construction of self means that DID - essentially a dysfunction of self - must be understood as a response to overwhelming, usually traumatic, experiences that are necessarily shaped by cultural norms and behavioural repertoires of the context in which it occurs. In African, Asian, and other non-Western countries - where social constructions of self are relatively porous to influences external to the person - DID usually takes the form of pathological possession experiences which are more congruent with a conception of self as not separate or individual (Cardeña et al., 2009). Thus research in India (Chaturvedi et al., 2010), Japan (Umesue et al., 1996), Oman (Chand et al., 2000), China (Xiao, et al., 2006), and Iran (Alvi and Assad, 2011) has found a high prevalence of DD (>5\%), but few, if any, 'non-possession-form' DID cases.

This situation may change with inclusion in the DSM-5 (APA, 2013) of presentations characterised by pathological possession in the diagnostic criteria for DID: "Disruption of identity characterized by two or more distinct personality states, which may be described in some cultures as an experience of possession" (p. 292). This diagnostic broadening will likely increase the validity of DID criteria cross-culturally, making the description of the disorder more consonant with cultural constructions of self that are inter-dependent and patterned by religious beliefs about spiritual beings. In such settings, pathological fragmentation of self is expressed in the idiom of external malicious forces that disrupt identity and consciousness. There is not, however, a strict dichotomy between Western and non-Western expressions, as a subgroup of patients with DID in North America and Turkey attribute the origin of at least some of their identities 
to spirit possession (Ross, 2011; Şar et al., 1996). Nor is pathological possession

exclusively associated with DID. It represents instead a behavioural "final common pathway" (Carr and Vitaliano, 1985) that is normative in many cultures (though not "normal" when it causes distress) and can present as part of many disorders, not only DID (e.g., how depressive symptoms present in disorders beyond mood disorders). DSM5 refers to the presentation of pathological possession in individuals with DID, but does not equate all pathological possession with this disorder.

Alongside cultural factors, data have consistently shown that DID is associated with traumatic and stressful experiences. Large-scale clinical and epidemiological studies in the United States, Australia, Turkey, Puerto Rico, and Canada have found that DID is linked to antecedent severe, chronic abusive and traumatic experiences in childhood, typically at the hands of an attachment figure (e.g., Martínez-Taboas, 1991; Middleton and Butler, 1998; Ross et al. 1990b; Şar, 2011). Dalenberg et al. (2012) calculated Ross and Ness' (2010) comparison of DID patients to controls, and found effect sizes of .74.78 for physical and sexual abuse. More severe and earlier-onset child abuse appears to differentiate DID from other disorders (Boon and Draijer, 1993b). By using corroborating documentation from hospital, police and child protection agencies or witnesses, several studies confirm histories of severe abuse in DID (Coons, 1994; Martínez-Taboas, 1991; Lewis et al., 1997). Studies exploring DID as a longitudinal outcome of confirmed child abuse are needed to examine further the abuse-DID link.

Every study that has systematically examined aetiology has found that antecedent severe, chronic childhood trauma is present in the histories of almost all individuals with DID. Yet the interplay between trauma and DID in non-Western countries (Asia, Africa, 
Arabia) has been understudied. Ugandan villagers with pathological possession had more psychoform and somatoform dissociation, and had suffered greater traumatic exposure, than randomly selected mentally healthy inhabitants from the same village (Van Duijl et al., 2010). However, research in Turkey suggests that milder presentations of DID are sometimes associated with traumatisation that is covert, such as severely dysfunctional communication and relationship styles in family members (Öztürk and Şar, 2005).

Understanding the aetiology of DID requires the amalgamation of several exposure-, coping- and developmental factors. These include traumatic experiences, family dynamics, child development, attachment (Kluft, 1993; Putnam, 2006; Ross, 1997) and the role culture plays in constructing "alternate" selves (i.e. embodied representations of the metaphor of "a different person" [or spiritual being]) with separate attributes and specific memories for trauma). DID develops when a child is exposed to chaos, coercion, and most commonly, overt severe physical and/or sexual abuse, often with disorganised attachment to caregivers. The child must also have the biological capacity to dissociate to an extreme level, leading to multiple states that do not become integrated over time. Such self-states allow the child to compartmentalise overwhelming and conflicting feelings of betrayal, terror, love and shame (Putnam, 2006; Van der Hart et al., 2006). Overwhelmed by intense conflicting needs and emotions, the child is unable to integrate discrete behavioural and emotional states into a coherent or relatively integrated self according to the appropriate socio-cultural construction/s of self (Putnam, 2006). In certain (e.g., mainstream Western) cultures, this process is consonant with a fragmentation of internal identities; in other (e.g., non-Western) cultures it may accord with external spiritual entities that take control of the individual's consciousness and 
identity. In summary, existing data demonstrate that development of DID is likely due to a complex combination of traumatic experiences, dissociative processes, psychosocial mediators and socially constructed understandings of self.

\section{Epidemiology of DID}

The absence of DD modules in diagnostic interviews assessing general psychopathology (e.g., SCID, CIDI; First et al., 1997; World Health Organization, 1997), may account for the lack of DID data from large-scale community-based epidemiological studies (Andrews et al., 2001; Bijl et al., 1998). Measures such as the SCID-D and DDIS have been developed to assess the epidemiology of DID.

\section{Clinical Studies}

Findings from consecutive samples of inpatients and outpatients in general psychiatric clinics in diverse countries vary by clinical setting, and to some extent geographic region. Two cross-sectional studies from North America found that 4.0-5.4\% of psychiatric inpatients met DSM-IV criteria for DID (Ross et al., 1991; Saxe et al., 1993). In Turkey, the prevalence rate of DID is $5.4 \%$ among general psychiatric inpatients, 2.8\% among substance dependence inpatients, and 2.0-2.5\% among general psychiatric outpatients (Karadag et al., 2005; Şar et al., 2003; Tutkun et al., 1998). Inpatient rates are 2.0\% in the Netherlands (Friedl and Draijer, 2000), 0.9\% in Germany (Gast et al., 2001), and 0.4\% in Switzerland (Modestin et al., 1996). The highest prevalence is seen in psychiatry emergency departments or outpatient units that receive emergency admissions. For example, cross-sectional rates were $14.0 \%$ in a university emergency department in Istanbul (Şar et al., 2007) and 6.0\% in an outpatient psychiatric unit in New York City that included emergency admissions (Foote et al., 2006). 
Marked variation in prevalence $(0.4 \%-14.0 \%)$ is likely due, at least in part, to methodological differences across studies and settings (Friedl et al., 2000). Research using the semistructured SCID-D usually reports lower rates of DID than the fullystructured DDIS. Since the SCID-D requires clinicians to judge which experiences are dissociative in nature, use of the SCID-D may lead to exclusion of more false positive cases than the DDIS. Other explanations for the variation may be cultural factors which influence both emergence of DID and interpretation of symptoms (Şar et al., 2013). For example, European studies report substantially lower rates of DID than Turkish or North American studies. While each European country may be relatively homogenous in sociocultural factors influencing identity formation, North America and Turkey may be characterised by more dramatic cultural diversity.

Overall, cross-sectional prevalence of DID tends to increase with level of psychiatric severity, ranging from about $2 \%$ in outpatient clinics to about $5 \%$ in inpatient units, with even higher rates in emergency settings.

\section{Community Studies}

Community-based epidemiological studies describe the full extent and distribution of the disorder in the population. This is because clinical epidemiology research is affected by local utilisation patterns for mental health services, as determined by accessibility factors and variations in the severity and impairment associated with the disorder (Fleming and Hsieh, 2002). Unfortunately, community-based research on DID is limited. One representative sample from Manitoba, Canada found a lifetime prevalence of $3.1 \%$ for DID using the DDIS and DSM-III-R criteria (Ross, 1991). A representative 
DID: An empirical overview

sample of women in Sivas City, Turkey $(\mathrm{N}=648)$ had a lifetime prevalence of $1.1 \%$ using the DSM-IV version of the DDIS (Şar, Akyuz and Dogan, 2007).

For practical reasons, proxy instruments may be used to estimate diagnostic rates. A community-based epidemiological study in New York State ( $\mathrm{N}=658)$ used the DESTaxon items for initial screening. Four SCID-D items ( 2 on dissociative amnesia and 2 on identity alteration) were then administered to approximate a DID diagnosis (Johnson et al., 2006). Results yielded a 12-month prevalence for DID of $1.5 \%$. While the findings in Sivas City and New York State produced similar rates of DID, the prevalence in Manitoba was higher, due to utilisation of the DSM-III-R criteria which did not list amnesia among diagnostic criteria of DID.

Epidemiological studies of DID have utilised DSM-III-R or DSM-IV diagnostic criteria. The DSM-5 introduced specific forms of pathological possession into the DID criteria. A recent general population study of 628 Turkish women (Sar et al., in press) found two of the 13 with an experience of possession had DID (seven women in the sample had DID). The diagnostic heterogeneity of the pathological possession experiences is consistent with the "final common pathway" concept of possession in DSM-5. Yet most epidemiological studies do not distinguish possession trance that meet DID criteria from that which does not. In India, for example, the prevalence of trance and possession disorders was reported at 5.3\% among inpatients and $11.5 \%$ among outpatients in a tertiary referral psychiatric hospital over a 10-year period (Chaturvedi et al., 2010). However, no diagnosis of DID was made. This is likely to change when DSM5 criteria are used in future studies. 
DID: An empirical overview

\section{Psychobiological Findings Related to DID}

Unique neurophysiological profile of DID

Although imaging studies have elucidated neurophysiological markers of the dissociative response in patients with a range of DD and PTSD, studies performed specifically in DID patients are more circumscribed. Different imaging techniques support three as yet unintegrated hypotheses. On the whole, single photon emission computerised tomography (SPECT) studies support an orbitofrontal hypothesis; magnetic resonance imaging (MRI), functional MRI (fMRI) and positron emission tomography (PET) studies support a cortico-limbic hypothesis; and EEG and QEEG studies support a temporal hypothesis for DID.

Forrest (2001) proposed a neurodevelopmental model for DID, underlining deficient functionality of the orbitofrontal region in the brain. The orbitofrontal lobe has been hypothesised to be affected by early trauma. Consistent with this orbitofrontal hypothesis, DID patients exhibited orbitofrontal hypoperfusion in comparison with normal controls in two SPECT studies (Şar et al., 2001; Şar et al., 2007) conducted in "host" identities (i.e., identities predominantly engaging with the external world). Bilaterally increased perfusion in medial and superior frontal regions and occipital areas accompanied orbito-(inferior) frontal hypoperfusion in one of these studies (Şar et al., 2007). There was no difference in perfusion of any brain area between different identities (Şar et al., 2001).

With respect to the cortico-limbic hypothesis as originally formulated in the context of PTSD studies (Lanius et al., 2006), a structural MRI study established that DID patients have smaller hippocampi and amygdalae than normal controls (Vermetten 
et al., 2006). Ehling et al. (2007) also found reduced volumes in the parahippocampal gyrus of individuals with DID and strong correlations between reduction of parahippocampal volume and both psychoform and somatoform dissociation.

Moreover, significant functional brain imaging (PET and fMRI) differences have been found between (1) different identities in DID patients (Reinders et al., 2003, 2006; Schlumpf et al., 2013) and (2) perfusion before versus perfusion during "switching" between identities in a DID patient (Tsai et al., 1999). In the PET studies by Reinders et al. an "emotional" dissociative identity (associated with trauma memories), when compared to an "apparently normal" dissociative identity (numb and depersonalised from trauma memories), showed increased cerebral blood flow in the amygdala, insular cortex, and somatosensory areas in the parietal cortex and the basal ganglia, as well as certain areas in the occipital and parietal cortex and anterior cingulate and frontal areas (Reinders et al., 2003, 2006). In a subsequent PET study, healthy controls simulating two identity states were unable to reproduce the same network patterns as DID patients (Reinders et al., 2012).

In the fMRI study by Tsai et al. (1999) bilateral hippocampal inhibition, right parahippocampal and medial temporal inhibition, and inhibition in small regions of the substantia nigra and globus pallidus were seen during the switch into another identity, as well as right hippocampal activation when the participant was returning to the original identity. The fMRI studies by Wolk and coworkers (Savoy et al., 2012; Wolk et al., 2012) demonstrate activation of the primary sensory and motor cortices, frontal and prefrontal regions and nucleus accumbens during switching in a DID patient. In summary, the switching process in DID is typified by activation and inhibition of a varying array of 
neurological areas and structures. The exact patterning of these may be related to the psychobiological characteristics of the dissociative identities involved.

Electrophysiological differences between identity states have also been found in a DID patient, who after 15 years of diagnosed cortical blindness, gradually regained sight during psychotherapeutic treatment. Waldvogel et al. (2007) demonstrated absent visual evoked potentials (VEP) in the blind identity versus normal VEP in the seeing identity. As a neural basis of such psychogenic blindness, the authors assumed a top-down modulation of activity in the primary visual pathway, possibly at the level of the thalamus or the primary visual cortex.

The temporal hypothesis of DID is supported by conventional visual EEG studies (Coons et al., 1988; Mesulam, 1981) as well as some quantitative EEG (QEEG) studies. In the QEEG study by Lapointe et al. (2006), variability between identity states involved mostly beta activity in the frontal and temporal lobes. On the other hand, Cocker et al. (1994) reported increased frontal QEEG delta activity in the hypnotically-induced "baby" identity in a patient with DID. A QEEG brain mapping study by Hughes et al. (1990) demonstrated left temporal and posterior-temporal-occipital changes in the theta and beta-2 frequency range in four of 11 identities in a DID patient. Further partial support for the temporal hypothesis comes from Hopper et al. (2002) who demonstrated that the average alpha coherence on QEEG was lower for 'alter' identities than for "host" identities in five DID patients in some temporal, frontal, parietal and central regions.

The temporal hypothesis is also supported by some SPECT studies. Saxe et al. (1992) demonstrated increased activation in the left temporal lobe in four assessed identities of a DID patient. In Şar et al.'s (2001) SPECT study the "host" identity showed 
increased perfusion in the left (dominant hemisphere) lateral temporal region compared to healthy controls. This lateralisation was not replicated in a follow-up study (Şar, Unal and Öztürk, 2007).

Imaging and neurophysiological studies have shown discrete brain areas of interest in understanding DID. No studies that failed to support any of these hypotheses were found, and it is not clear whether the three hypotheses are competing. The specific areas identified may reflect technical aspects of the specific methods. For example, notwithstanding the EEG's excellent temporal resolution, it has limited spatial resolution, which might explain its lack of findings on the deeper brain structures and hence its noncontribution to the other two hypotheses.

Future empirical studies using combinations of imaging methodologies specifically in DID might shed light on the relationship between and a possible merging of the orbitofrontal, cortico-limbic and temporal hypotheses, as well as a possible amalgamation of these neurophysiological findings with the findings of cognitive psychological studies.

\section{Cognitive correlates of DID}

The cognitive study of DID is emerging from diagnostic, empirical and anecdotal evidence of memory, attention and information processing anomalies associated with the disorder (e.g., APA, 2013; Dorahy and Huntjens, 2007). Some scientific consideration has been given to attention and working memory processes in DID (Stringer and Cooley, 1994). Results are beginning to suggest a cognitive architecture supporting vigilance and bias for threat stimuli, the nature of which may vary depending on the psychological 
characteristics of the dissociative identity assessed (e.g., Dale et al., 2008; Dorahy, Middleton, and Irwin, 2005; Hermans et al., 2006).

A limited number of studies have examined encoding and retrieval processes within dissociative identities. Case studies show some evidence of generalised childhood amnesia (Schacter et al., 1989) and that memory retrieval seemingly differs across identities (Bryant, 1995). After the incipient dissolution of amnesia, traumatic childhood memories may return initially as sensorimotor fragments (e.g., images, body sensations) rather than as a verbal narrative among adults with DID (Van der Hart et al., 2005). There are empirical suggestions that within-identity encoding and retrieval may differ for fear versus neutral stimuli, with fear stimuli less effectively encoded (Barlow, 2011). Retrieval appears to be better for 'gist' information than for specific details (Barlow, 2011). This suggests the yet-to-be assessed possibility that DID might be characterised by overgeneralised memory (non-specific retrieval) especially for fear narratives, as has been found in other disorders (e.g., depression, PTSD; see Moore and Zoellner, 2007).

The bulk of contemporary research on cognition in DID has focused on the specific nature of information compartmentalisation (i.e., the isolation of material within a dissociative identity) and transfer (i.e., the transmission of material across dissociative identities; Allen and Iacono, 2001; Dorahy, 2001b). This follows the lead of early investigations (Prince and Peterson, 1909) and is associated with the well-documented apparent amnesia between some dissociative identities for cognitive representations of experience. Research has largely focused on procedural, perceptual and nonautobiographical semantic and episodic information transfer (e.g., Eich et al., 1997; Nissen et al., 1988; Peters et al., 1998). 
Increasing methodological sophistication addressing concerns with external and ecological validity is beginning to question some of the findings from previous work that showed evidence of compartmentalisation (especially for more complex information, such as stories that contained considerable contextual information). For example, in a reaction time study of nine DID patients, Huntjens et al. (2012) found evidence of semantic autobiographical transfer across dissociative identities, despite participants reporting amnesia between identities. Participants provided answers to autobiographical semantic questions (e.g., names of siblings) in two amnestic identities. Two weeks later, participants were presented word lists interspersed with autobiographical but irrelevant words and previously learned target words. They were required to rapidly identify target words. It was hypothesised that the reaction time for autobiographically-salient words would be slower than that for non-autobiographical words (including the autobiographically-salient words of the other identity). However, the DID sample showed the same slower response to words in both identities' word lists, suggesting that semantic autobiographical information is not compartmentalised despite being experienced as such. The pattern of findings was not replicated in controls or individuals simulating DID.

These findings, as well as other studies (e.g., Huntjens et al., 2003; Kong et al., 2008), challenge complete compartmentalisation of information. In short, research indicates that dissociative amnesia operates at a metacognitive level, such that the person subjectively experiences alterations in memory retrieval between identities that are not verified in the laboratory (i.e., the person believes they are unaware of the information, 
perhaps due to major alterations in conscious faculties, despite laboratory evidence to the contrary).

Thus amnesia in DID appears to operate in the same (metacognitive) way as many symptoms in other disorders (e.g., perceived fatness in anorexia, perceived danger or amnesia in PTSD, perceived catastrophe in panic disorder). In terms of phenomenology as well as aetiology, amnesia in DID can be likened to conversion symptoms. Both amnesia and conversion in DID are functional neurological symptoms. Similarly, sensorimotor functions vary between identities in DID without structural neurological pathology, yet functional neurobiological variations are found (Bhuvaneswar and Spiegel, 2013).

Future research should explore the nature and mechanisms of amnesia, as well as elucidating the nature of attentional processes in DID. Emotionally-charged episodic autobiographical memory transfer across functionally amnesic identities likewise merits thorough investigation.

\section{Treatment of DID}

DID treatment outcome has been systematically studied for three decades via case studies, case series, cost-efficacy studies, and naturalistic outcome studies with followups as long as 10 years (e.g., Coons and Bowman, 2001; Coons and Sterne, 1986; Kluft, 1984). Research indicates that therapy utilising a phasic trauma treatment model consistent with expert consensus guidelines is beneficial to DID individuals (Brand et al., 2009b; International Society for the Study of Trauma and Dissociation [ISSTD], 2011). A meta-analysis of eight non-controlled DD studies found pre/post within-participant effect sizes in the medium-to-large range for outcomes including improved dissociation, 
anxiety, distress, and depression (Brand et al., 2009b). Treatment was associated with reductions in diagnoses of comorbid axis I and II disorders, suicidality, and substance abuse; improvements were maintained at two-year follow-up (Brand et al., 2009b; Ellason and Ross, 1997).

The phasic model of DID treatment involves patients working towards establishing safety and stability in Stage 1. Some DID patients may lack interest in, and/or the psychological or practical resources for, moving beyond Stage 1. In Stage 2, the focus is on maintaining stability while exploring trauma narratives and resolving trauma-related emotions, beliefs, and behaviours. In Stage 3, the treatment emphasises integration of identities and living without reliance on dissociation. A survey of international DID experts coalesced in recommending interventions to be used with DID patients across the stages of treatment (Brand et al., 2012). Stabilising safety and containment of traumatic material were highly endorsed in all but the last stage of treatment. Core interventions recommended at every stage of treatment included: providing psychoeducation; increasing awareness and tolerance of emotion; developing impulse control; fostering grounding skills to manage dissociation; nurturing the therapeutic alliance; and managing stressors, current relationships and daily functioning. The consistency between these experts' recommendations, those described in the ISSTD Treatment Guidelines (2011), and the interventions documented in the Treatment of Patients with Dissociative Disorders (TOP DD) study (Brand et al., 2009a) suggest that a standard of care for the treatment of DID is emerging. Detailed discussions of DID treatment are also available (e.g., Boon et al., 2011; Chu, 2011; Howell, 2011; ISSTD, 2011). 
Case studies have yielded critical insights into the treatment of DID (Kluft, 1984). One of the most rigorous - a single case experimental design - demonstrated that cognitive analytic treatment resulted in statistically and clinically significant improvements that remained stable or continued to increase over six months of follow-up for a woman with DID (Kellett, 2005). The patient also showed sudden improvements following targeted interventions, indicating that the treatment was central to the improvements.

The longitudinal, international TOP DD study is providing new understanding of DID treatment. The TOP DD study prospectively assessed treatment response from 230 DID patients and their therapists from 19 countries, across four data collection points over 30 months (Brand et al., 2009b; Brand et al., 2013). Over time, patients showed statistically significant reductions in dissociation, PTSD, distress, depression, hospitalisations, suicide attempts, self-harm, dangerous behaviours, drug use, and physical pain, as well as higher Global Assessment of Functioning scores (Brand et al., 2013).

Even participants with the highest levels of dissociation and the most severe depression showed improvement over time (Engelberg and Brand, 2013; Stadnik and Brand, 2013). Younger patients stabilised self-destructive and suicidal behaviours more rapidly than older patients, suggesting the importance of early diagnosis and treatment (Myrick et al., 2012). Rates of revictimisation showed a trend towards reduction, and more patients showed "sudden improvement" than "sudden worsening" (i.e., 20\% decrease or increase in symptoms, respectively; Myrick et al., 2013). Those who suddenly improved had fewer revictimisations and stressors compared to those who 
worsened. Only $1.1 \%$ of patients showed worsening over more than one data collection point; , a rate that compares favourably to the $5 \%-10 \%$ of general patients who show worsening symptoms during treatment (Hansen et al., 2002). The consistency of statistical improvement across a range of symptoms and adaptive functioning, strongly suggests that treatment contributed to improvements.

It is important to consider health costs associated with DID. A Canadian treatment study of DID concluded that annual costs dropped from CAD $\$ 75,000$ to CAD $\$ 36,000$ (Canadian) in the three years after treatment for DID (Ross and Dua, 1993). This and other studies document considerable cost savings even for those who had been chronically ill before being appropriately treated for DID (Lloyd, 2011).

In summary, research indicates that DID treatment consistent with the standard of care outlined in the expert guidelines for this disorder is associated with improvements in functioning and reduction of a wide range of symptoms. Although randomised trials are difficult to conduct with DID patients due to their symptom complexity and high suicidality, current evidence suggests that DID treatment accounts for documented improvements. Studies using systematic treatment with blind assessments are critically needed to identify how to treat these patients most effectively. Trials could be developed that compare individual treatment alone to individual treatment plus manualised DID group therapy or web-based psychoeducational interventions.

\section{Conclusion}

The empirical literature on DID emerging over the past 30 years shows that, beyond the rhetoric and controversy, DID is a valid disorder characterised by amnesia, identity confusion, and coexistence of dissociative identities which can be differentiated 
from other psychiatric disorders as well as from feigned presentations of DID.

Characteristic features include a complex array of co-existing symptoms associated with psychosis, mood, anxiety, affect regulation and personality functioning. A mix of subtle and overt developmental, interpersonal and cultural drivers produce DID, with childhood attachment-based trauma appearing to be a universal factor, while social idioms of self produce components of cultural specificity. DID is found around the globe in almost every culture in which researchers have carefully assessed for the range of dissociative symptoms.

Orbitofrontal, cortico-limbic and temporal anomalies are evident in DID, with different neurobiological profiles found across identities than those in simulation. Cognitive functioning, while varying across identities, appears to support biases in threat detection and management. Reported amnesia between identities may be produced by metacognitive processes, but studies are yet to assess transfer of autobiographical episodic memories for traumatic events. Despite the complexity of DID at neurobiological, cognitive, relational and symptomatic levels, assessment and treatment consistent with the expert consensus guidelines for this disorder (ISSTD, 2011) have produced consistently positive results. The challenge of randomised, well-controlled intervention protocols awaits empirical investigation.

While empirical research on DID accumulates, a diverse collection of challenges impact the development of studies and acceptance of their findings and implications. At issue are not only matters of 'science', but the psychological and social challenges of assimilating and responding to what science comprises. A convergence of contextual issues relates as much to challenges to existing paradigms as to the principles of scientific 
inquiry. DID questions the concept of self as an autonomous and integrated entity, and thus challenges understanding of the nature of scientific enquiry itself. However, it is clear that research on DID is proceeding and advancing, providing fascinating insights into the power of the mind to cope with developmental trauma and severe attachment disruptions in the cultural contexts in which they occur.

Declaration of Conflicting Interests:

The Authors declare that there is no conflict of interest

\section{References}

Alvi T and Assad F (2011) Presentation of psychiatric co-morbidity in patients presenting with dissociative disorders. Journal of Pakistani Psychiatric Society 8(1): 18-21.

Allen JJB and Iacono WG (2001) Assessing the Validity of Amnesia in Dissociative Identity Disorder: A Dilemma for the DSM and the Courts. Psychology, Public Policy and Law 7(2): 311-344.

American Psychiatric Association (1980) Diagnostic and Statistical Manual of Mental Disorders (3th ed.). Washington, DC: Author.

American Psychiatric Association (2013) Diagnostic and Statistical Manual of Mental Disorders (5th ed). Arlington, VA: Author.

Anderson-Fye EP and Becker AE (2004) Sociocultural aspects of eating disorders. In Thompson JK (ed) Handbook of Eating Disorders and Obesity. New Jersey: Wiley, pp.565-589. 
Andrews G, Henderson S and Hall W (2001) Prevalence, comorbidity, disability and service utilisation. Overview of the Australian National Mental Health Survey. British Journal of Psychiatry 178: 145-153.

Barlow MR (2011) Memory for complex emotional material in dissociative identity disorder. Journal of Trauma and Dissociation 12(1): 53-66.

Bernstein EM and Putnam FW (1986) Development, reliability and validity of a dissociation scale. Journal of Nervous and Mental Disease 174(12): 727735.

Bhuvaneswar C and Spiegel D (2013) An eye for an I: A 35-year-old woman with fluctuating oculomotor deficits and dissociative identity disorder. International Journal of Clinical and Experimental Hypnosis 61(3): 351-370.

Bijl RV, Ravelli A and Van Zessen B (1998) Prevalence of psychiatric disorder in the general population: Results of the Netherlands Mental Health Survey and Incidence Study (NEMESIS). Social Psychiatry Psychiatric Epidemiology 33(12): $587-595$.

Boon S and Draijer N (1991) Diagnosing dissociative disorders in the Netherlands: A pilot study with the structured clinical interview for DSM-III-R dissociative disorders. American journal of Psychiatry 148(4): 458-462.

Boon S and Draijer N (1993a) Multiple personality disorder in The Netherlands: A clinical investigation of 71 patients. American Journal of Psychiatry 150(3): 489494.

Boon S and Draijer N (1993b) The differentiation of patients with MPD or DDNOS from patients with a cluster B personality disorder. Dissociation 6(2/3): 126-135. 
DID: An empirical overview

Boon S, Steele K and Van der Hart O (2011) Coping with Trauma-Related Dissociation: Skills Training for Patients and Therapists. New York: Norton.

Brand BL, Armstrong JG, Loewenstein RJ et al. (2009c) Personality differences on the Rorschach of dissociative identity disorder, borderline personality disorder, and psychotic inpatients. Psychological Trauma: Theory, Research, Practice, and Policy 1(3): 188-205.

Brand BL and Chasson GS (in press) Distinguishing Simulated from Genuine Dissociative Identity Disorder on the MMPI-2. Psychological Trauma: Theory, Research, Practice, \& Policy.

Brand BL, Classen CC, Lanius RA et al. (2009a) A naturalistic study of dissociative identity disorder and dissociative disorder not otherwise specified patients treated by community clinicians. Psychological Trauma: Theory, Research, Practice \& Policy 1(2):153-171.

Brand BL, Classen C, McNary SW et al. (2009b) A review of dissociative disorders treatment studies. Journal of Nervous and Mental Disease 197(9):646-654

Brand BL, McNary SW, Loewenstein RJ et al. (2006) Assessment of genuine and simulated Dissociative Identity Disorder on the Structured Interview of Reported Symptoms. Journal of Trauma \& Dissociation 7(1): 63-85.

Brand BL, McNary SW, Myrick AC et al. (2013) A longitudinal, naturalistic study of dissociative disorder patients treated by community clinicians. Psychological Trauma: Theory, Research, Practice \& Policy 5(4): 301-308.

Brand BL, Myrick AC, Loewenstein RJ et al. (2012) A survey of practices and 
DID: An empirical overview

recommended treatment interventions among expert therapists treating patients with dissociative identity disorder and dissociative disorder not otherwise specified. Psychological Trauma: Theory, Research, Practice \& Policy 4(5): 490500.

Brand BL, Stadnik R and Savoca A (2013) Personality Assessment Inventory profile and predictors of elevations among dissociative disorder patients. Journal of Trauma \& Dissociation 14(5): 546-561.

Brand BL, Tursich, M, Tzall, D, \& Loewenstein, RJ (in press). Utility of the SIRS-2 in Distinguishing Genuine From Simulated Dissociative Identity Disorder, Psychological Trauma: Theory, Research, Practice, \& Policy.

Bryant RA (1995) Autobiographical memory across personalities in dissociative identity disorder: A case report. Journal of Abnormal Psychology 104(4): 625-631.

Butcher JN, Graham JR, Ben-Porath YS et al. (2001) Manual for the Administration and Scoring of the MMPI-2. Minneapolis: University of Minnesota Press.

Cardeña E, Van Duijl M, Weiner LA and Terhune DB (2009) Possession/trance phenomena. In Dell PF and O'Neil JA (eds) Dissociation and the Dissociative Disorders. New York: Routledge, pp.171-181.

Carr JE and Vitaliano PP (1985) The theoretical implications of converging research on depression and the culture-bound syndromes. In Kleinman A and Good B (eds) Culture and Depression: Studies in the Anthropology and Cross-Cultural Psychiatry of Affect and Disorder. Berkeley: University of California Press, pp.244-266. 
Castillo R (1997) Culture and Mental Illness. CA: Brooks/Cole.

Chand SP, Al Hussaini AA, Martin R et al. (2000). Dissociative disorders in the Sultanate of Oman. Acta Psychiatrica Scandinavica 102(3): 185-187.

Chaturvedi SK, Desia G and Shaligram D (2010) Dissociative disorders in a psychiatric institute in India - A selected review and patterns over a decade. International Journal of Social Psychiatry 56(5): 533-539.

Chu JA (2011) Rebuilding Shattered Lives: Treating Complex PTSD and Dissociative Disorders ( $2^{\text {nd }}$ edition). New York: Wiley.

Cocker KI, Edwards GA, Anderson JW and Meares RA (1994) Electrophysiological changes under hypnosis in multiple personality disorder: A two-case exploratory study. Australian Journal of Clinical and Experimental Hypnosis 22(2): 165-76.

Coons PM (1994) Confirmation of childhood abuse in childhood and adolescent cases of multiple personality disorder and dissociative disorder not otherwise specified. Journal of Nervous and Mental Disease 182(8): 461-464.

Coons PM and Bowman ES (2001) Ten-year follow-up study of patients with dissociative identity disorder. Journal of Trauma \& Dissociation 2(1): 73-89.

Coons PM, Bowman ES and Milstein V (1988) Multiple personality disorder: A clinical investigation of 50 cases. Journal of Nervous and Mental Disease 176(9): 519527.

Coons PM and Sterne AL (1986) Initial and follow-up psychological testing on a group of patients with multiple personality disorder. Psychological Reports 58(1): 839845.

Cross SE and Markus HR (1999) The cultural constitution of personality. In Pervin L and 
DID: An empirical overview

John O (eds) Handbook of Personality: Theory and Research. New York:

Guilford, pp.378-396.

Dale KY, Flaten MA, Elden $\AA$ and Holte A (2008) Dissociative identity disorder and prepulse inhibition of the acoustic startle reflex. Neuropsychiatric Disease and Treatment 4(3): 653-662.

Dalenberg CJ, Brand B L, Gleaves DH et al. (2012) Evaluation of the evidence for the trauma and fantasy models of dissociation. Psychological Bulletin 138(3): 550588.

Dell PF (2006) The Multidimensional Inventory of Dissociation (MID): A comprehensive measure of pathological dissociation. Journal of Trauma \& Dissociation 7(2): 77-106.

Dorahy MJ (2001a) Culture, cognition and dissociative identity disorder. In Schumaker JF and Ward T (eds) Culture, Cognition and Psychopathology. Westport, Conn: Praeger, pp.157-169.

Dorahy MJ (2001b) Dissociative identity disorder and memory dysfunction: The current state of experimental research, and its future directions. Clinical Psychology Review 21(5): 771-795.

Dorahy MJ and Huntjens R (2007) Memory and attentional processes in dissociative identity disorder: A review of the empirical literature. In Vermetten E, Dorahy MJ and Spiegel D (eds) Traumatic Dissociation: Neurobiology and Treatment. Arlington, VA: American Psychiatric Publishing, Inc, pp.55-75.

Dorahy MJ, Middleton W, and Irwin HJ (2005) The effect of emotional context on cognitive inhibition and attentional processing in dissociative identity 
disorder. Behaviour Research and Therapy 43(5): 555-568.

Dorahy MJ, Shannon C, Seagar L et al. (2009) Auditory hallucinations in dissociative identity disorder and schizophrenia with and without a childhood trauma history: similarities and differences. Journal of Nervous and Mental Disease 197(12): 892-898.

Draijer N and Boon S (1999) The imitation of dissociative identity disorder: Patients at risk, therapists at risk. Journal of Psychiatry \& Law 27: 423-458.

Ehling T, Nijenhuis ERS and Krikke AP (2007) Volume of discrete brain structures in complex dissociative disorders: Preliminary findings. In de Kloet ER, Oitzl MS and Vermetten E (eds) Progress in Brain Research 167: 307-310.

Eich E, Macauley D, Loewenstein RJ et al. (1997) Memory, amnesia, and dissociative identity disorder. Psychological Science 8(6): 417-422.

Ellason JW and Ross CA (1997) Two-year follow-up of inpatients with dissociative identity disorder. American Journal of Psychiatry 154(6): 832-839.

Ellason JW, Ross CA, and Fuchs DL (1996) Lifetime Axis I and II comorbidity and childhood trauma history in dissociative identity disorder. Psychiatry 59(3): 255266.

Engelberg J and Brand BL (2012) The effects of depression on self-harm and treatment outcome in patients with severe dissociative disorder. Psi Chi Journal of Psychological Research, 17(3), 115-124.

Eshun S and Gurung RAR (Eds) (2009) Culture \& Mental Health: Sociocultural Influences on Mental Health. Malden, MA: Blackwell.

Fink D, and Golinkoff M (1990) MPD, borderline personality disorder and 
DID: An empirical overview

schizophrenia: A comparative study of clinical features. Dissociation 3(3): 127134.

First MB, Spitzer RL Gibbon M and Williams JB (1997) Structured Clinical Interview for DSM-IV Axis I Disorders (SCID-I), Clinician Version, User's Guide, Washington DC: American Psychiatric Association.

Fleming JA, and Hsieh CC (2002) Introduction to epidemiologic research methods. In Tsuang MT and Tohen M (ed) Textbook of Psychiatric Epidemiology $\left(3^{\text {rd }}\right.$ ed). New York: Wiley, pp. 3-34.

Foote B, Smolin Y, Kaplan M et al. (2006) Prevalence of dissociative disorders in psychiatric outpatients. American Journal of Psychiatry 163(4): 623-629.

Forrest KA (2001) Toward an etiology of dissociative identity disorder: A neurodevelopmental approach. Consciousness and Cognition 10(3): 259-293.

Friedl MC and Draijer N (2000). Dissociative disorders in Dutch Psychiatric inpatients. American Journal of Psychiatry 157(6): 1012-1013.

Friedl MC, Draijer N, and De Jonge P (2000) Prevalence of dissociative disorders in psychiatric in-patients: The impact of study characteristics. Acta Psychiatrica Scandinavica 102(6): 423-428.

Gast U, Rodewald F, Nickel V and Emrich HM (2001) Prevalence of dissociative disorders among psychiatric inpatients in a German university clinic. Journal of Nervous and Mental Disease 189(4): 249-257.

Gingrich H (2009) Assessing dissociative symptoms and dissociative disorders in college students in the Philippines. Journal of Aggression, Maltreatment and Trauma 18(4): 403-418. Doi: 10.1080/10926770902901881 
DID: An empirical overview

Gleaves DH (1996) The sociocognitive model of dissociative identity disorder: A reexamination of the evidence. Psychological Bulletin 120(1): 42-59

Gleaves DH, May M and Cardeña E (2001) An examination of the diagnostic validity of dissociative identity disorder. Clinical Psychology Review 21(4): 577-608.

Hansen NB, Lambert MJ, and Forman EM (2002) The psychotherapy dose-response effect and its implications for treatment delivery services. Clinical Psychology: Science and Practice 9(3): 329-343.

Herman J (1992) Trauma and Recovery. New York: Basic Books.

Hermans EJ, Nijenhuis ERS, Van Honk J et al. (2006) Identity state-dependent attentional bias for facial threat in dissociative identity disorder. Psychiatry Research 141(2): 233-236.

Honig A, Romme MAJ, Ensink BJ et al. (1998) Auditory hallucinations: A comparison between patients and nonpatients. Journal of Nervous and Mental Disease 186(10): 646-651.

Hopper A, Ciorciari J, Johnson G et al. (2002) EEG coherence and dissociative identity disorder. Journal of Trauma \& Dissociation 3(1): 75-88.

Hornstein, G. (2013) Whose account matters? Feminism and Psychology, 23(1), 29-40.

Horovitz RP and Braun BG (1984) Are multiple personalities borderline? An analysis of 33 cases. Psychiatric Clinics of North America 7(1): 69-87.

Howell EF (2011). Understanding and Treating Dissociative Identity Disorder. Routledge: New York.

Hughes JR, Kuhlman DT, Fichtner CG et al. (1990) Brain mapping in a case of multiple personality. Clinical Electroencephalography 21(4): 200-9. 
Huntjens RJC, Postma A, Peters ML et al. (2003) Interidentity amnesia for neutral, episodic information in dissociative identity disorder. Journal of Abnormal Psychology 112(2): 290-297.

Huntjens RJC, Verschuere B and McNally RJ (2012) Inter-identity autobiographical amnesia in patients with dissociative identity disorder. Plos One, 7(7): e40580. Doi: 10.1371/journal.pone.0040580

International Society for the Study of Dissociation (Chu, JA, Dell, PF, Somer, E et al.) (2011) Guidelines for treating dissociative identity disorder in adults, third revision. Journal of Trauma \& Dissociation 12(2): 115-87.

Janet P (1907) The Major Symptoms of Hysteria. London/New York: Macmillan. Reprint of 1920 edition: New York: Hafner, 1965.

Johnson JG, Cohen P, Kasen S and Brook JS (2006) Dissociative disorders among adults in the community, impaired functioning, and axis I and II comorbidity. Journal of Psychiatric Research 40(2): 131-140.

Karadag F, Şar V, Tamar-Gürol D et al. (2005) Dissociative disorders among inpatients with drug or alcohol dependency. Journal of Clinical Psychiatry 66(10): 1247-1253.

Kellett S (2005). The treatment of dissociative identity disorder with cognitive analytic therapy: Experimental evidence of sudden gains. Journal of Trauma \& Dissociation, 6(3): 55-81.

Kemp K, Gilbertson AD, and Torem M (1988) The differential diagnosis of multiple personality disorder from borderline personality disorder. Dissociation 1(4): 4146. 
Kluft RP (1984). Treatment of multiple personality disorder: A study of 33 cases.

Psychiatric Clinics of North America 7(1): 9-29.

Kluft RP (1987) First rank symptoms as a diagnostic clue to multiple personality disorder. American Journal of Psychiatry 144(3): 293-298.

Kluft RP (1993) Multiple personality disorder. In Spiegel D (ed) Dissociative Disorders: A Clinical Review. Lutherville, MD: Sidran Press, 17-44.

Kluft RP (1997) The argument for the reality of delayed recall of trauma. In Appelbaum PS Uyehara LA and Elin MR (eds) Trauma and Memory. New York: Oxford University Press, 25-57.

Kong LL, Allen JJB, and Glisky EL (2008) Interidentity memory transfer in dissociative identity disorder. Journal of Abnormal Psychology 117(3): 686-692.

Korman GP and Molina AI (2010) Cultura y depresión.[Culture and depression]. Argentina: Akadia Editorial.

Lanius RA, Bluhm R, Lanius U and Pain C (2006) A review of neuroimaging studies in PTSD: Heterogeneity of response to symptom provocation. Journal of Psychiatric Research 40(8): 709-729.

Lapointe AR, Crayton JW, DeVito R et al. (2006) Similar or disparate brain patterns? The intra-personal EEG variability of three women with multiple personality disorder. Clinical EEG and Neuroscience 37(3): 235-42.

Lewis DO, Yeager CA, Swica Y et al. (1997) Objective documentation of child abuse and dissociation in 12 murderers with dissociative identity disorder. American Journal of Psychiatry 154(12): 1703-1710.

Lewis-Fernández R, Hinton DE, Laria AJ et al. (2010) Culture and the anxiety disorders: 
Recommendations for DSM-V. Depression and Anxiety 27(2): 212-229.

Lewis-Fernández R, Martínez-Taboas A, Şar V et al. (2007) The cross-cultural assessment of dissociation. In Wilson JP and So-Kum Tang CC (eds) CrossCultural Assessment of Trauma and PTSD. New York: Springer, 279-317.

Lipsanen T, Korkeila J, Peltola P et al. (2004). Dissociative disorders among psychiatric patients: Comparison with a nonclinical sample. European Psychiatry 19: 53-55.

Lloyd M (2011) How investing in therapeutic services provides a clinical cost saving in the long term. Health Service Journal (online): http://tinyurl.com/74sefbz. Accessed 11/2012.

Markus HR and Kitayama S (2010) Cultures and selves: A cycle of mutual constitution. Perspectives on Psychological Science 5(4): 420-430.

Martínez-Taboas A (1991) Multiple personality in Puerto Rico: Analysis of fifteen cases. Dissociation: Progress in the Dissociative Disorders 4(4): 189-192.

McDowell DM, Levin FR and Nunes EV (1999) Dissociative identity disorder and substance abuse: The forgotten relationship. Journal of Psychoactive Drugs 31(1): 71-83.

McHugh P and Putnam F (1995) Resolved: multiple personality disorder is an individually and socially created artifact. Journal of the American Academy of Child and Adolescent Psychiatry 34: 957-962.

Merskey H (1992) The manufacture of personalities. The production of multiple personality disorder. The British Journal of Psychiatry 160: 327-340.

Mesulam M-M (1981) Dissociative states with abnormal temporal lobe EEG: Multiple personality and the illusion of possession. Archives of Neurology 38(3): 176-181. 
Middleton W and Butler J (1998) Dissociative identity disorder: An Australian series. Australia and New Zealand Journal of Psychiatry 32(6): 794-804.

Modestin J, Ebner G, Junghan M and Erni T (1996) Dissociative experiences and psychiatric disorders in acute psychiatric inpatients Comprehensive Psychiatry 37(5): 355-361.

Moore SA and Zoellner LA (2007) Overgeneral autobiographical memory and traumatic events: An evaluative review. Psychological Bulletin 133(3): 419-437.

Morey LC (1991) Personality Assessment Inventory: Professional Manual. Odessa, FL: Psychological Assessment Resources, Inc.

Mueller-Pfeiffer C, Rufibach K, Perron N et al. (2012) Global functioning and disability in dissociative disorders. Psychiatry Research 200(2/3): 475-481.

Mueller-Pfeiffer C, Rufibach K, Wyss D et al. (2013) Screening for dissociative disorders in psychiatric out- and day care-patients. Journal of Psychopathology and Behavioral Assessment. Doi: 10.1007/s10862-013-9367-0

Mulder RT (2012) Cultural aspects of personality disorder. In Widiger TA (ed) The Oxford Handbook of Personality Disorders. New York: Oxford University Press, pp.260-274.

Myrick AC, Brand BL, McNary SW et al. (2012) An Exploration of Young Adults' Progress in Treatment for Dissociative Disorder. Journal of Trauma \& Dissociation 13(5): 582-595.

Myrick AC, Brand BL and Putnam FW (2013) For better or worse: the role of revictimization and stress in the course of treatment for dissociative disorders. Journal of Trauma \& Dissociation 14(4): 375-389. 
DID: An empirical overview

Nijenhuis ERS, Van Dyck R, Spinhoven P et al. (1999). Somatoform dissociation discriminates among diagnostic categories over and above general psychopathology. Australian and New Zealand of Journal of Psychiatry 33(4): $511-520$.

Nissen MJ, Ross JL, Willingham DB et al. (1988) Memory and awareness in a patient with multiple personality disorder. Brain and Cognition 8(1): 117-134.

Öztürk E and Şar V (2005) 'Apparently normal' family: A contemporary agent of transgenerational trauma and dissociation. Journal of Trauma Practice 4(3-4): 287-303.

Peters ML, Uyterlinde SA, Consemulder J et al. (1998) Apparent amnesia on experimental memory tests in dissociative identity disorder. Consciousness and Cognition 7(1): 27-41.

Prince M (1905) The Dissociation of a Personality. New York: Longmans, Green. Reprint: Greenwood Press, New York, 1969.

Prince M, and Peterson F (1909) Experiments in psychogalvanic reactions from coconscious ideal in a case of multiple personality. Journal of Abnormal Psychology 3: 114-131.

Putnam FW (2006) Dissociative disorders. In Cicchetti D and Cohen DJ (eds) Developmental Psychopathology. New York: Wiley, Vol. II, pp.657-695.

Putnam FW, Carlson EB, Ross CA et al. (1996). Patterns of dissociation in clinical and nonclinical samples. Journal of Nervous and Mental Disease 184(11): 673-679.

Reinders AATS, Nijenhuis ERS, Paans AMJ et al. (2003) One brain, two selves. NeuroImage 20(4): 2119-2125. 
DID: An empirical overview

Reinders AATS, Nijenhuis ERS, Quak J et al. (2006) Psychobiological characteristics of dissociative identity disorder: A symptom provocation study. Biological Psychiatry 60(7): 730-740.

Reinders AATS, Willemsen A, Vos H et al. (2012) Fact or factitious? A psychobiological study of authentic and simulated dissociative identity states. Plos One 7(6): e39279. doi:10.1371/journal.pone.0039279.

Rhoades GF and Şar V (eds) (2005) Trauma And Dissociation in a Cross-Cultural Perspective. Binghamton, NY: Haworth Press.

Robins E and Guze S (1970) Establishment of diagnostic validity in psychiatric illness: its application to schizophrenia. American Journal of Psychiatry 126(7): 983-987.

Rodewald F, Dell PF, Wilhelm-Gößling C et al. (2011a). Are major dissociative disorders characterized by a qualitatively different kind of dissociation. Journal of Trauma \& Dissociation 12(1): 9-24.

Rodewald F, Wilhelm-Gößling C, Emrich HM et al. (2011b). Axis-I comorbidity in female patients with dissociative identity disorder and dissociative identity disorder not otherwise specified. Journal of Nervous and Mental Disease 199(2): $122-131$.

Rogers R, Sewell KW, and Gillard ND (2010) Structured Interview of Reported Symptoms-2 (SIRS-2) and Professional Manual. Lutz, FL: Psychological Assessment Resources.

Ross CA (1991) Epidemiology of multiple personality disorder and dissociation. Psychiatric Clinics of North America 14(3): 503-517.

Ross CA (2004) Schizophrenia: Innovations in Diagnosis and Treatment. Birmingham, 
NY: Haworth Press.

Ross CA (1997) Dissociative Identity Disorder: Diagnosis, Clinical Features, and Treatment of Multiple Personality. New York: Wiley.

Ross CA (2011) Possession experiences in dissociative identity disorder: A preliminary study. Journal of Trauma \& Dissociation 12(4): 393-400.

Ross CA, Anderson G, Fleisher WP and Norton GR (1991) The frequency of multiple personality disorder among psychiatric inpatients. American Journal of Psychiatry 148(12): 1717-1720.

Ross C and Dua V (1993) Psychiatric health care costs of multiple personality disorder. American Journal of Psychotherapy 47(1): 103-112.

Ross CA, Duffy, CMM and Ellason JW (2002) Prevalence, reliability and validity of dissociative disorders in an inpatient setting. Journal of Trauma and Dissociation 3(1): 7-17.

Ross CA, Heber S, Norton GR et al. (1989a) Differences between multiple personality disorder and other diagnostic groups on structured Interview. Journal of Nervous and Mental Disease 177(8): 487-491.

Ross CA, Heber S, Norton GR et al. (1989b) The Dissociative Disorders Interview Schedule: a structured interview. Dissociation 2(3): 169-172.

Ross CA, Miller SD, Reagor P et al. (1990a) Schneiderian symptoms in multiple personality disorder and schizophrenia. Comprehensive Psychiatry 31(2): 111118. 
DID: An empirical overview

Ross CA, Miller SD, Reagor P et al. (1990b) Structured interview data on 102 cases of multiple personality disorder from four centers. American Journal of Psychiatry 147(5): 596-601.

Ross CA and Ness L (2010) Symptom patterns in dissociative identity disorder patients and the general population. Journal of Trauma \& Dissociation 11(4): 458-468.

Şar V (2011) Epidemiology of dissociative disorders: An overview. Epidemiology Research International doi:10.1155/2011/404538.

Şar V, Akyuz G and Dogan O (2007) Prevalence of dissociative disorders among women in the general population. Psychiatry Research 149(1-3): 169-176.

Şar V, Alioğlu F, Akyüz G (in press) Experiences of possession and paranormal phenomena among women in the general population: Are they related to traumatic stress and dissociation? Journal of Trauma and Dissociation.

Şar V, Krüger C, Martinez-Taboas A et al. (2013) Sociocognitive and posttraumatic models of dissociation are not opposed. Journal of Nervous and Mental Disease 201(5): 439-440.

Şar V, Kundakci T, Kiziltan E et al. (2003) The Axis-I Dissociative Disorder Comorbidity of Borderline Personality Disorder Among Psychiatric Outpatients. Journal of Trauma \& Dissociation 4(1): 119-136.

Şar V, Koyuncu A and Öztürk E (2007) Dissociative disorders in the emergency psychiatric ward. General Hospital Psychiatry 29(1): 45-50.

Şar V and Öztürk E (2009) Psychotic presentations of dissociative identity disorder. In: Dell PF and O'Neil JA (eds) Dissociation and Dissociative Disorders:DSM-V and Beyond. New York: Routledge, pp.535-545. 
Şar V, Öztürk E, Islam S et al. (2012). Zwischen Selbstreflexion und Selbst-

Überzeugtsein: Kognitive Einsicht bei dissoziativen und schizophrenen Störungen und das Dissoziationsparadox. In: Özkan I, Sachsse U, and Streeck-Fischer A. (eds) Zeit heilt nicht alle Wunden: Kompendium zur Psychotraumatologie.

Göttingen: Vandenhoeck Ruprecht, pp.161-172.

Şar V and Ross CA (2006) Dissociative disorders as a confounding factor in psychiatric research. Psychiatric Clinics of North America 29(1): 129-144.

Şar V and Ross CA (2009) A research agenda for the dissociative disorders field. In Dell, PF and O'Neil JA (eds) Dissociation and the Dissociative Disorders: DSM-V and Beyond. New York: Routledge, pp.693-708.

Şar V, Taycan O, Bolat N et al. (2010). Childhood trauma and dissociation in schizophrenia. Psychopathology 43: 33-40.

Şar V, Unal SN, Kızıltan E et al. (2001) HMPAO SPECT study of cerebral perfusion in dissociative identity disorder. Journal of Trauma \& Dissociation 2(2): 5-25.

Şar V, Unal SN and Öztürk E (2007) Frontal and occipital perfusion changes in dissociative identity disorder. Psychiatry Research-Neuroimaging 156(3): 217 223.

Şar V, Yargiç LI and Tutkun H (1996) Structured interview data on 35 cases of dissociative identity disorder in Turkey. American Journal of Psychiatry 153(10): $1329-1333$.

Savoy RL, Frederick BB, Keuroghlian AS et al. (2012) Voluntary switching between identities in dissociative identity disorder: A functional MRI case study. Cognitive Neuroscience 3(2): 112-119. 
Saxe GN, Van der Kolk BA, Berkowitz R et al. (1993) Dissociative disorders in psychiatric inpatients. American Journal of Psychiatry 150(7): 1037-1042.

Saxe GN, Vasile RG, Hill TC et al. (1992). SPECT imaging and multiple personality disorder. The Journal of Nervous and Mental Disease 180(10): 662-663.

Schacter DL, Kihlstrom JF, Kihlstrom LC et al. (1989) Autobiographical memory in a case of multiple personality disorder. Journal of Abnormal Psychology 98(4): 508-514.

Schlumpf YR, Nijenhuis ERS, Chalavi S et al. (2013) Dissociative part-dependent biopsychosocial reactions to backward masked angry and neutral faces: An fMRI study of dissociative identity disorder. NeuroImage: Clinical 3: 54-64.

Spiegel D, Lewis-Fernández R, Lanius R et al. (2013) Dissociative disorders in DSM-5. Annual Review of Clinical Psychology 9: 299-326.

Stadnik R and Brand BL (2013). What contributes to predicting change in treatment of dissociation: Initial levels of dissociation, PTSD, or overall distress? Journal of Trauma \& Dissociation, 14(3), 328-341.

Stein DJ, Koenen KC, Friedman MJ et al. (2013) Dissociation in posttraumatic stress disorder: evidence from the world mental health surveys. Biological Psychiatry 73(4): 302-312

Steinberg M (1994a) Interviewer's guide to the Structured Clinical Interview for DSM-IV Dissociative Disorders - Revised (SCID-D-R) ( $2^{\text {nd }}$ ed.). Washington, DC: American Psychiatric Press.

Steinberg M (1994b) Structured Clinical Interview for DSM-IV Dissociative Disorders Revised (SCID-D-R) (2 $2^{\text {nd }}$ Ed.). Washington, DC: American Psychiatric Press. 
DID: An empirical overview

Steinberg M, Cicchetti D, Buchanan J et al. (1994) Distinguishing between multiple personality disorder (dissociative identity disorder) and schizophrenia using the structured clinical interview for DSM-IV dissociative disorders. Journal of Nervous and Mental Disease 182(9): 495-502.

Steinberg M, Rounsaville B and Cicchetti D (1990) The Structured Clinical Interview for DSM-III-R Dissociative Disorders: preliminary report on a new diagnostic instrument. American Journal of Psychiatry 147(1): 76-82.

Steinberg M, Rounsaville B and Cicchetti D (1991) Detection of dissociative disorders in psychiatric patients by a screening instrument and a structured diagnostic interview. The American Journal of Psychiatry 148(8): 1050-1054.

Stompe T and Friedmann A (2007) Culture and schizophrenia. In Bhugra D and Bhui K (eds) Textbook of Cultural Psychiatry. New York: Cambridge University Press, pp.314-322.

Stringer AY and Cooley EL (1994) Divided attention performance in multiple personality disorder. Neuropsychiatry, Neuropsychology, \& Behavioral Neurology 7(1): 51-56

Taylor E (1983) William James on Exceptional Mental States: The 1896 Lowell Lectures. Amherst: MA: The University of Massachusetts Press.

Tsai GE, Condie D, Wu MT and Chang IW (1999) Functional magnetic resonance imaging of personality switches in a woman with dissociative identity disorder. Harvard Review of Psychiatry 7(2): 119-122.

Tutkun H, Şar V, Yargiç LI et al. (1998) Frequency of dissociative disorders among 
DID: An empirical overview

psychiatric inpatients in a Turkish university clinic. American Journal of Psychiatry 155(6): 800-805.

Tutkun H, Yargiç LI and Şar V (1995). Dissociative identity disorder: A clinical investigation of 20 cases in Turkey. Dissociation 8(1): 3-9.

Umesue M, Matsuo T, Iwata N and Tashiro N (1996) Dissociative disorders in Japan: a pilot study with the Dissociative Experiences Scale and a semistructured interview. Dissociation 9: 182-189.

Van der Hart O, Bolt H and Van der Kolk BA (2005) Memory fragmentation in dissociative identity disorder. Journal of Trauma and Dissociation 6(1): 55-70.

Van der Hart O, Nijenhuis ERS and Steele K (2006) The Haunted Self: Structural Dissociation and the Treatment of Chronic Traumatization. New York: Norton. Van der Hart O, Witztum E and Friedman B (1993) From hysterical psychosis to reactive dissociative psychosis. Journal of Traumatic Stress 6(1): 43-64.

Van Duijl M, Nijenhuis E, Komproe IH et al. (2010). Dissociative symptoms and reported trauma among patients with spirit possession and matched healthy controls in Uganda. Culture, Medicine \& Psychiatry, 34(2), 380-400.

Vermetten E, Schmahl C, Lindner S et al. (2006). Hippocampal and Amygdalar Volumes in Dissociative Identity Disorder. American Journal of Psychiatry 163(4): 630-636.

Waldvogel B, Ulrich A, and Strasburger H (2007) Sighted and blind in one person. Nervenarzt 78: 1303-1309.

Waller NG, Putnam FW and Carlson EB (1996) Types of dissociation and dissociative types: A taxometric analysis of dissociative experiences. Psychological Methods 
1(3): 300-321.

Welburn KR, Fraser GA, Jordan SA et al. (2003) Discriminating dissociative identity disorder form schizophrenia and feigned dissociation on psychological tests and structured interview. Journal of Trauma \& Dissociation 4(2): 109-130.

World Health Organisation (1993) The ICD-10, Classification Of Mental And Behavioural Disorders. Geneva: Author.

World Health Organization (1997) Composite International Diagnostic Interview Version 2.1, Geneva,World Health Organization.

Wolk PC, Savoy RL and Frederick BB (2012) The neural correlates of vertical splitting in a single case study. Neuropsychoanalysis 14(2): 157-163.

Xiao Z, Yan H, Wang Z, Zou Z et al. (2006) Trauma and dissociation in China. American Journal of Psychiatry 163(8): 1388-1391. 